Original Research Paper

\title{
Gene Expression Profiling of Toll-Like Receptor 4 and 5 in Peripheral Blood Mononuclear Cells of Patients with Systemic Sclerosis
}

\author{
${ }^{1}$ Simin Almasi, ${ }^{2}$ Saeed Aslani, ${ }^{1}$ Hadi Poormoghim, \\ ${ }^{2}$ Ahmadreza Jamshidi, ${ }^{2}$ Shiva Poursani and ${ }^{2}$ Mahdi Mahmoudi \\ ${ }^{1}$ Firouzgar Hospital, Iran University of Medical Sciences (IUMS), Tehran, Iran \\ ${ }^{2}$ Rheumatology Research Center, Tehran University of Medical Sceinces, Tehran, Iran
}

Article history

Received: 30-12-2015

Revised: $15-03-2016$

Accepted: 17-03-2016

Corresponding Author: Mahdi Mahmoudi

Rheumatology Research

Center, Tehran University of

Medical Sceinces, Tehran, Iran

Tel: +98-21-8822-0067,

PO-BOX: 1411713137

Email: mahmoudim@tums.ac.ir

\begin{abstract}
The Toll-Like Receptor (TLR) family is appeared to be expressed in many cell types in the immune system and plays a role in the pathogenesis of various autoimmune diseases. The expression profile and role of TLRs in Systemic Sclerosis (SSc) have been partly explained. It is aimed through this investigation to evaluate the expression pattern of TLR 4 and 5 in Peripheral Blood Mononuclear Cells (PBMCs) from SSc patients. PBMCs were isolated from whole blood of $20 \mathrm{SSc}$ patients and 50 healthy individuals. Total RNA content of leukocytes was extracted. Then, cDNA was synthesized from the mRNA of the cells. Afterward, Quantitative analysis was carried out through Real-Time PCR using the TaqMan Gene Expression Assays. An over expression of TLR5 mRNA in PBMCs from SSc patients was seen in comparison to healthy individuals. Nevertheless, the gene expression of TLR4 in SSc patients remained almost equal to controls. Our findings suggest that over expression of TLR5 in SSc patients may be involved in the pathogenesis of SSc.
\end{abstract}

Keywords: Scleroderma, TLR4, TLR5, PBMC, Gene Expression

\section{Introduction}

Systemic Sclerosis (SSc) is a systemic connective tissue disease, characterized by fibrosis of the skin and internal organs, injuries of the small arteries (microangiopathy) and the presence of autoantibodies (Varga and Abraham, 2007). A pathogenic role in the SSc has been suggested through interactions between modified endothelial cells and cells of immune system in eliciting changed fibroblast functional (Jimenez and Derk, 2004). Furthermore, interplay between environmental factors and genetic modification is thought to be contributing factor in the SSc (Tan and Arnett, 2000). Fibroblasts in SS are distinguished by high amount of collagen synthesis and excess fibrotic condition over degradation is thought to take place (Pattanaik et al., 2015).

Toll-Like Receptors (TLRs) are a group of type I integral membrane glycoproteins, which almost 11 families of them have been described in human (Botos et al., 2011). TLRs play an essential role in the innate immune system as Pathogen-Recognition Receptors (PRRs) to recognize conserved structures within microbes known as Pathogen-Associated Molecular Patterns (PAMPs) and self-cells derived Damage-Associated Molecular Patterns (DAMPs) (Janeway Jr, 1992). Several DAMPs in distinct pathogens are recognized by TLRs that leads to a signaling cascade and results in production of various pro-inflammatory cytokines, chemokines and type I IFNs (Akira et al., 2006).

Regarding to several autoimmune diseases, endogenous ligands or pathogens-activated immune cells and even fibroblasts and endothelial cells in SSc, have become more plausible to have role in such disease. Circulating TLR4 ligands have been identified in serum of SSc patients (Roelofs et al., 2005). Ligation of TLR4 can induce an increased production of IL-10 in monocyte-derived Dendritic Cells (moDCs) from SSc patients. Taking interleukin (IL)-10 production is related with the increased level of profibrotic CC Chemokine Ligand (CCL)-18, a profibrotic role for TLR-activated moDCs can be implied (van Lieshout et al., 2009). TLR4 has been shown to play a role in SSc patients and monocytes from SSc patients with interstitial lung disease have an enhanced pro-fibrotic phenotype. These 
monocytes can differentiate into fibrocytes and secrete collagen in higher amounts after exposure to the LPS, the TLR4 agonist (Mathai et al., 2010). Moreover, activation of fibroblast TLR4 signaling, triggered by damage-associated endogenous TLR4 ligands, results in increased transforming growth factor- $\beta 1$ sensitivity with increased matrix production and progressive connective tissue remodeling. Accordingly, fibroblast TLR4 serves as the switch for converting self-limited tissue repair into intractable fibrosis (Bhattacharyya et al., 2013). On the other side, TLR5 shows increment in SSc fibroblasts and may have a suppressive impression on collagen expression. Presumably, the over expression of TLR5 in SSc fibroblasts is a negative response against tissue fibrosis (Sakoguchi et al., 2013).

TLRs pathway and overactivity, especially TLR4, have been related to SSc immunopathogenesis through involvement in secretion of chemokines and cytokines. Furthermore, the over expression of TLR4 and 5 genes among patients with SSc has already been observed. Given the before evidences, in this study we aimed to assess expression levels of TLR4 and 5 in Peripheral Blood Mononuclear Cells (PBMCs), of SSc patients as a confirmatory investigation of expression profile of these molecules.

\section{Subjects and Methods}

\section{Patients and Healthy Control Subjects}

The patients were recruited from Firouzgar Hospital Rheumatology Department and diagnosed as having systemic sclerosis according to the American College of Rheumatology criteria for SSc (LeRoy et al., 1988). Twenty SSc patients and 50 healthy age- and sexmatched healthy volunteers were included in the study (Table 1). Patients had received no immunomodulatory therapy for at least 3 months before they were included in the study. Moreover, SSc patients who were suspicious of other plausible autoimmune disorders were excluded. None of the healthy controls had autoimmune diseases in neither themselves nor their immediate family members. The Human Research Ethics Committees from the Tehran University of Medical Sciences approved this study. Written informed consent was taken by all participants. Blood samples from SSc patients were obtained during clinical diagnosis; as such, samples were obtained from healthy controls. About $5 \mathrm{~mL}$ of blood from each subject was collected in EDTA-anticoagulated tubes and ESR blood collection test tubes using venipuncture.

\section{PBMC Preparation and RNA Extraction}

Ficoll-Hypaque density gradient centrifugation approach was used in order to isolate PBMCs from peripheral blood of the subjects. Total cellular RNA was extracted using High Pure RNA Isolation Kit (Roche, Germany) according to manufacturer's manuals. Yield and purity of RNA were determined using a NanoDrop spectrophotometer at 260/280 nm (Nano Drop ND-2000 C Spectrophotometer, Thermo Fisher Scientific, USA).

\section{Reverse Transcription and Complementary DNA Synthesis}

Complementary DNA (cDNA) was synthesized from the RNA of cells using the Transcriptor First Strand cDNA Synthesis Kit (Roche, Germany) according to the manufacturer's instruction. Concisely, first $4 \mu \mathrm{L}$ of isolated RNA $(30 \mu \mathrm{g})$ through previous procedure were mixed with $1 \mu \mathrm{L}$ of random hexamer primer and $7 \mu \mathrm{L}$ RNASe-free $\mathrm{H}_{2} \mathrm{O}$ and then incubated at $65^{\circ} \mathrm{C}$ for $5 \mathrm{~min}$. Afterwards, micro-tubes were chilled on ice and a mixture of reaction buffer $4 \mu \mathrm{L}$, RNASe inhibitor $1 \mu \mathrm{L}$, dNTP mix $2 \mu \mathrm{L}$ and reverse transcriptase $1 \mu \mathrm{L}$ was added to each sample. Samples were immediately incubated at $25^{\circ} \mathrm{C}$ for $5 \mathrm{~min}$ followed by $42^{\circ} \mathrm{C}$ for 60 min; the reaction was finally terminated by heating at $70^{\circ} \mathrm{C}$ for $5 \mathrm{~min}$. Reverse transcription was performed with the final volume of $20 \mu \mathrm{L}$ pre tube.

\section{Real-Time Quantitative Polymerase Chain Reaction}

Quantitative analysis was carried out by Real Time PCR using the TaqMan Gene Expression Assays containing the FAM dye-labeled probes (Taq Man Predesigned Gene Expression Products, Applied Bio systems, Foster City, CA, USA) and StepOnePlus RealTime PCR System (Applied Bio systems, Foster City, CA, USA). Each reaction mixture contained a total volume of $20 \mu \mathrm{L}$ (master mix $10 \mu \mathrm{L}$, cDNA $2 \mu \mathrm{L}$ [5 ng mL], assay mix $1 \mu \mathrm{L}$ and $\mathrm{H}_{2} \mathrm{O} 7 \mu \mathrm{L}$ ). The Real Time PCR conditions were: $50^{\circ} \mathrm{C}$ for $2 \mathrm{~min}, 95^{\circ} \mathrm{C}$ for 10 min, then 40 cycles of $95^{\circ} \mathrm{C}$ for $15 \mathrm{sec}$ and $60^{\circ} \mathrm{C}$ for 1 min. A widely used approach to represent relative gene expression, the comparative $\mathrm{C}_{\mathrm{T}}$ method, was exploited to evaluate expression as previously described by (Schmittgen and Livak, 2008). Relative amounts of target mRNAs in test samples were calculated and normalized to the corresponding Betaactin (ACTB) mRNA transcript level as a housekeeping gene. Then relative expression for each sample was calculated using the following equation: Relative mRNA expression $=\left(2^{-\Delta \mathrm{Ct}}\right) \times 10^{3}$.

\section{Statistical Analysis}

Data analysis was fulfilled via SPSS software version 18 (SPSS, Chicago, IL, USA). Scale variables were calculated for normality using the Kolmogorov-Smirnov test. Through the independent sample $t$-test, group 
comparisons of continues variables were carried out. If the variable was not normally distributed, MannWhitney nonparametric test was conducted. The GraphPad Prism version 5.00 for Windows (GraphPad Software, La Jolla California USA, www.graphpad.com) was applied to illustrate data through graph. All results are expressed as mean \pm Standard Error of Mean (SEM) with statistical significance set at 5\%.

\section{Results}

Demographic traits of SSc patients are listed in the Table 1. Case subjects with the mean age of $40.7 \pm 6.9$ were found to be age matched with healthy control group with that of $42 \pm 11.5$ ( $>00.05)$. The participants were also sex matched and of the $20 \mathrm{SSc}$ patients, $15(75 \%)$ and 5 $(25 \%)$ were male and female respectively; healthy subjects were consisted of 37 (74\%) males and $13(26 \%)$ females. A significant difference $(p<0.001)$ was observed between ESR values of SSc patients $(21.26 \pm 16.1)$ and healthy individuals $(4.4 \pm 3.82)$.

No significant difference was observed (fold change $=1.11 ; p=0.34)$ in the expression level of TLR4 between SSc patients and healthy controls (Fig. 1A). Nevertheless, PBMCs from SSc patients expressed the TLR5 mRNA highly (2.49 times up regulated; $p=$ 0.011 ) in comparison to healthy subjects (Fig. 1B).

Table 1. Baseline Specifications of SSc patients $(\mathrm{N}=20)$

\begin{tabular}{|c|c|c|}
\hline Characteristic & Mean $(\%)$ & Standard deviation \\
\hline Age & 42 & 11.5 \\
\hline \multicolumn{3}{|l|}{ Gender } \\
\hline Male & $15(75)$ & - \\
\hline Female & $5(25)$ & - \\
\hline Disease duration & 7.05 & 7.77 \\
\hline \multicolumn{3}{|l|}{ Disease subset } \\
\hline Limited & $14(70)$ & - \\
\hline Diffuse & $6(30)$ & - \\
\hline modified Rodnan Skin Score (mRSS) & 9.5 & 6.6 \\
\hline Forced Vital Capacity (FVC) & 2.5 & 0.9 \\
\hline Diffusing Capacity for Carbon Monoxide (DLCO) & 6.5 & 2.8 \\
\hline \multicolumn{3}{|l|}{ Fibrosis } \\
\hline Yes & $3(15)$ & - \\
\hline No & $17(85)$ & - \\
\hline human Placental Alkaline Phosphatase (hPAP) & 30.5 & 7.9 \\
\hline \multicolumn{3}{|l|}{ Pulmonary Hypertension (PHT) } \\
\hline Yes & $4(20)$ & - \\
\hline No & $16(80)$ & - \\
\hline \multicolumn{3}{|l|}{ Muscle weakness } \\
\hline Yes & $2(10)$ & - \\
\hline No & $18(90)$ & - \\
\hline \multicolumn{3}{|l|}{ Myosis } \\
\hline Yes & $1(5)$ & - \\
\hline No & $19(95)$ & - \\
\hline \multicolumn{3}{|l|}{ Gastrointestinal (GI) involvement } \\
\hline Yes & $16(80)$ & - \\
\hline No & $4(20)$ & - \\
\hline \multicolumn{3}{|l|}{ Alveolit } \\
\hline Yes & $1(5)$ & - \\
\hline No & $19(95)$ & - \\
\hline \multicolumn{3}{|l|}{ Pulmonary Arterial Hypertension (PAH) } \\
\hline No & $15(75)$ & - \\
\hline Primary & $4(20)$ & - \\
\hline Secondary & $1(5)$ & - \\
\hline \multicolumn{3}{|l|}{ Kidney involvement } \\
\hline Yes & $3(15)$ & - \\
\hline No & $17(85)$ & - \\
\hline \multicolumn{3}{|l|}{ Fluorescent Antinuclear Antibodies (FANA) } \\
\hline Positive & $16(80)$ & - \\
\hline Negative & $4(20)$ & - \\
\hline Anti-Centromere Antibodies (ACA) Titer & 15.7 & 6 \\
\hline \multicolumn{3}{|l|}{ Anti-topo I (Anti-DNA topoisomerase I) } \\
\hline Positive & $10(50)$ & - \\
\hline Negative & $10(50)$ & - \\
\hline Erythrocyte Sedimentation Rate (ESR) & 21.26 & 16.1 \\
\hline
\end{tabular}


Table 2. The correlations of the expressions of TLR4 and TLR5 mRNAs in PBMCs fromSSc patients with clinical manifestations

\begin{tabular}{|c|c|c|c|c|}
\hline \multirow[b]{2}{*}{ Parameter } & \multicolumn{2}{|l|}{ TLR4 } & \multicolumn{2}{|l|}{ TLR5 } \\
\hline & $r$ & $P$ value & $r$ & $P$ value \\
\hline Age & -0.214 & 0.379 & 0.171 & 0.485 \\
\hline ESR & 0.244 & 0.314 & 0.275 & 0.255 \\
\hline mRSS & 0.345 & 0.747 & 0.125 & 0.845 \\
\hline FVC & 0.225 & 0221 & 0.227 & 0.350 \\
\hline DLCO & 0101 & 0.681 & -0.112 & 0.653 \\
\hline hPAP & -0.017 & 0.946 & -0.103 & 0.596 \\
\hline ACA Titer & -0.210 & 0.387 & -0.175 & 0.474 \\
\hline
\end{tabular}

Toll-Like Receptor (TLR), Erythrocyte Sedimentation Rate (ESR), r: Pearson's Correlation Coefficient, Modified Rodnan Skin Score (mRSS), Forced Vital Capacity (FVC), Diffusing Capacity for Carbon Monoxide (DLCO), human Placental Alkaline Phosphatase (hPAP), Anti-Centromere Antibodies (ACA)

A

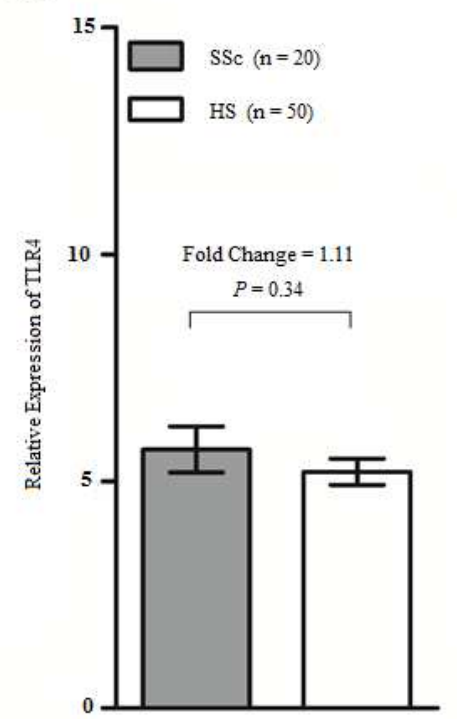

B

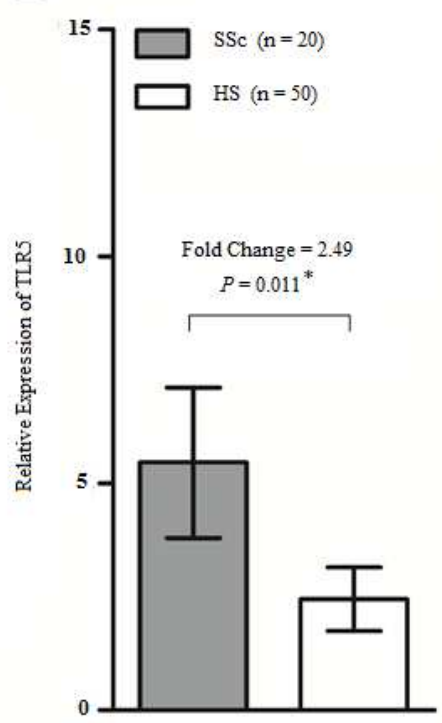

Fig. 1. Relative expression of TLR4 (A) and TLR5 (B) mRNA in PBMCs from SSc patients and healthy subjects is illustrated through Bar graph (SSc: Systemic Sclerosis, HS: Healthy Subjects)

A

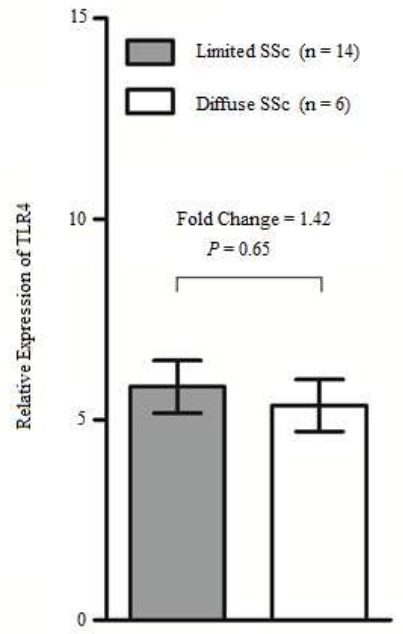

B

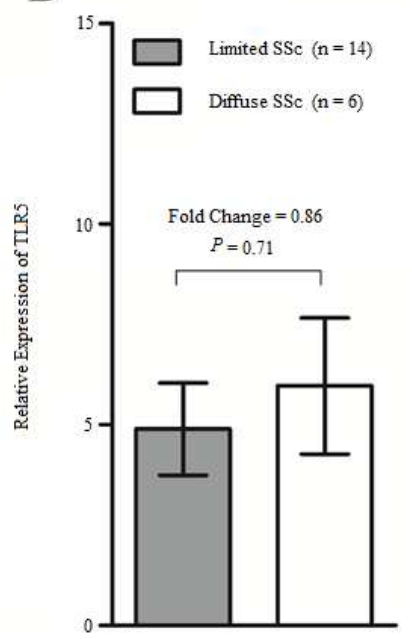

Fig. 2. Relative expression of TLR4 (A) and TLR5 (B) mRNA in PBMCs from Limited and Diffuse SSc patients is illustrated through Bar graph (SSc: Systemic Sclerosis) 
B

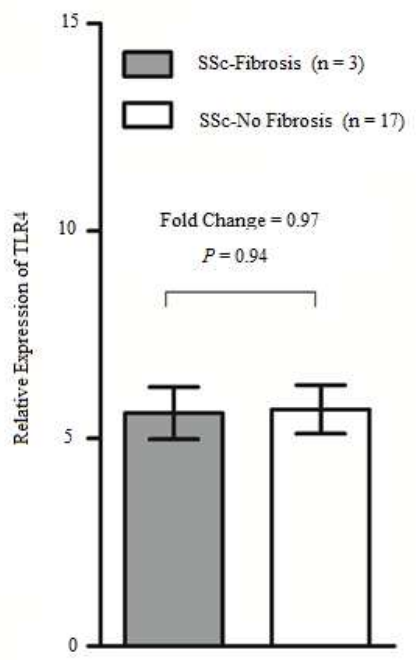

A

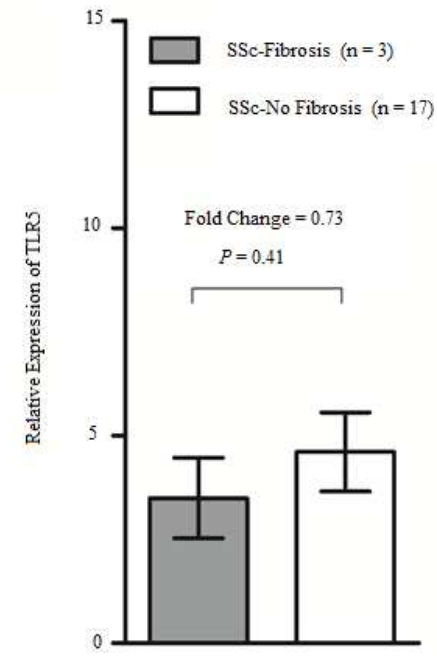

Fig. 3. Relative expression of TLR4 (A) and TLR5 (B) mRNA in PBMCs from SSc patients with and without Fibrosisis illustrated through Bar graph (SSc: Systemic Sclerosis)

Limited subset SSc patients expressed TLR4 higher (fold change $=1.42, p=0.65)$ than diffuse subset, but expressed TLR5 lower (fold change $=0.86, p=0.71$; Fig. 2). Both TLR4 and 5 were down regulated (Fig. 3) in SSc patients with fibrosis compared with patients without fibrosis but this differences were not statistically significant (fold change $=0.97 ; p=0.94$, fold change $=$ $0.73 ; p=0.41$, respectively).

Clinical specifications of age, ESR, mRSS, FVC, DLCO, hPAP and ACA titer of SSc patients correlated with neither TLR4 nor TLR5 mRNA expression level (Table 2).

\section{Discussion}

Current concentrations have been drawn toward the notion that systemic immune dysregulation is the central incident that ultimately may lead to fibroblast activation and development of SSc. Toll-like receptors are main molecules of innate immune system which recognize various DAMPs and PAMPs and relate them to adaptive immune system. A thorough immune response needs the interaction between both innate and adaptive immune systems and revolves around TLRs for activation of several pathways.

Outstanding clinical characteristics of systemic sclerosis are progressive fibrosis of the skin and internal organs. In the pathological point of view, SSc presents core manifestations are inflammation and autoimmunity, excessive deposition of Extracellular Matrix (ECM) and vasculopathy of small arteries (Varga and Abraham, 2007). Generally, the ECM contains fibrillins, collagens, proteoglycans, matrix molecules and fibroblasts and, myofibroblasts, which are pivotal effect or cells in development and pathogenesis of SSc (Varga, 2007). Transforming growth factor $\beta$ (TGF- $\beta$ ) can be produced and secreted by both resident and infiltrating cells in the dermis and can activate fibroblasts and induce differentiation into myofibroblasts (Kissin et al., 2006; Tomasek et al., 2002). The myofibroblasts, in turn, produce even more ECM, causing fibrosis and alongside with dermal fibroblasts secret various inflammatory cytokines and chemokines, like IL-6 and CCL-2 which are important inflammatory mediators in the pathogenesis of SSc (Feghali et al., 1994; Distler et al., 2001). Early skin biopsies of SSc patients show infiltration of mononuclear inflammatory cells around the vessels. Mononuclear cells through involvement of PRRs secret cytokines and chemokines that lead to over recruiting of inflammatory cells and promoting of the ECM deposition (Fleischmajer et al., 1977).

Recent studies focused on patients with SSc have disclosed a dysregulation of type I interferon (IFN) pathways. The presence of a type I IFN signature in peripheral blood cells from patients with SSc has been described by expression profiling investigations (Tan et al., 2006). Type I IFNs play role in the regulation and modulation of the immune system. Furthermore, they can modify several processes in the immune system such as differentiation, survival, proliferation and cytokine production of various immune cells. One of the immunoregulatory functions of IFNs is to stimulate immune cells to express the TLRs on themselves. TLRs are a group of Pattern Recognition Receptors (PRRs) in the innate immune system that sense common molecular patterns found on microbes and then initiate key inflammatory responses (Takeda and Akira, 2004). TLR activation can also trigger production and secretion of 
various inflammatory mediators like type I IFNs. It has been demonstrated that the interaction between TLRs and IFNs plays a role in the pathogenesis of autoimmune diseases, such as systemic lupus erythematous, which shows overlapping autoantibody specificities and sometimes overlapping clinical manifestations with SSc (Crow et al., 2003; Hua et al., 2006).

Fineschi et al. (2008) demonstrated that autoantibodies in SSc patients, which bind to the surface of fibroblasts, might contribute to the pathogenesis of SSc. They concluded that fibroblasts-binding autoantibodies, through up regulation of production of profibrotic and proangiogenic chemokines, in a proteasome and TLR4-dependent approach, play a role in SSc development (Fineschi et al., 2008). Van Lieshout et al. (2009) observed an increased serum level of CCL18 in SSc patients compared with healthy controls and this chemokine, which was mediated, at least partly, via IL-10 after TLR4 stimulation, was secreted by monocyte derived dendritic cells. The ongoing study investigated expression level of TLR4 mRNA in the PBMCs of SSc patients. Interestingly, we observed that TLR4 was not expressed differently in SSc patients compared with healthy subjects. Perhaps TLR4 on the specific cells other than a general immune cell population of PBMCs, such as monocyte derived dendritic cells and fibroblasts, contributes to the development of SSc.

Sakoguchi et al. (2013) investigated the expression profiles of TLR-related genes in SSc fibroblasts. They disclosed that TLR5 and TLR10 expressions were increased in SSc fibroblasts both in vitro and in vivo. Furthermore, they noted that TLR5 itself might have a suppressive impression on collagen expression and perhaps the over expression of TLR5 in SSc fibroblasts was the negative feedback against tissue fibrosis (Sakoguchi et al., 2013). To concord with the evidence, in this investigation the expression level of TLR5 was realized to be 2.49 times up regulated in PBMCs from SSc patients compared with healthy individuals. However, probable impression and function of increased expression level of TLR4 in PBMCs from SSc patients need further studies.

Even though SSc patients over expressed TLR5 mRNA, ESR values did not correlate with expression level of TLR5 mRNA. This prematurely imply to the note that, despite increased expression of TLR5 suppresses collagen expression, this increment do not affect inflammatory situation according to ESR level. Furthermore, no correlation was observed between other parameters, namely Age, mRSS, FVC, DLCO, hPAP and ACA titer and TLR5 expression level.

\section{Conclusion}

The conclusion to be reached is that, our results imply that dysregulation of TLR5 expression, but not
TLR4, may be associated with disease development in SSc patients. Hopefully, based upon beneficial effect of TLR5 increased expression in suppression of collagen production and increased ECM deposition, further studies will provide new strategies for TLR pathway intervention drug development to be therapeutic approaches for SSc patients.

\section{Acknowledgement}

The authors would like to express their gratitude to all participants who made the completion of this study possible. This study was supported by a research grant from Deputy of Research, Iran University of Medical Sciences.

\section{Author's Contributions}

Simin Almasi: Conception and design the experiments, acquisition of data, drafting the article and final approve.

Saeed Aslani: Analysis and interpretation of data, drafting the article, critical review, final approve.

Hadi Poormoghim: Acquisition of data, critical review and final approve.

Ahmadreza Jamshidi: Acquisition of data, critical review and final approve.

Shiva Poursani: Acquisition of data, analysis and interpretation of data, drafting the article, final approve.

Mahdi Mahmoudi: Conception and design the experiments, analysis and interpretation of data, drafting the article, final approve, critical review and final approve.

\section{Disclosure of Conflict of Interest}

The authors declare that they have no financial or other conflicts of interest in relation to this research and its publication.

\section{References}

Akira, S., S. Uematsu and O. Takeuchi, 2006. Pathogen recognition and innate immunity. Cell, 124: 783-801. DOI: 10.1016/j.cell.2006.02.015

Bhattacharyya, S., K. Kelley, D.S. Melichian, Z. Tamaki and F. Fang et al., 2013. Toll-like receptor 4 signaling augments transforming growth factor- $\beta$ responses: A novel mechanism for maintaining and amplifying fibrosis in scleroderma. Am. J. Pathol., 182: 192-205. DOI: 10.1016/j.ajpath.2012.09.007

Botos, I., D.M. Segal and D.R. Davies, 2011. The structural biology of Toll-like receptors. Structure, 19: 447-459. DOI: 10.1016/j.str.2011.02.004

Crow, M.K., K.A. Kirou and J. Wohlgemuth, 2003. Microarray analysis of interferon-regulated genes in SLE. Autoimmunity, 36: 481-490. DOI: $10.1080 / 08916930310001625952$ 
Distler, O., T. Pap, O. Kowal-Bielecka, R. Meyringer and S. Guiducci et al., 2001. Overexpression of monocyte chemoattractant protein 1 in systemic sclerosis: Role of platelet-derived growth factor and effects on monocyte chemotaxis and collagen synthesis. Arthritis Rheumatism, 44: 2665-2678. DOI: $\quad 10.1002 / 1529-0131(200111) 44: 11<2665:: A I D-$ ART446>3.0.CO;2-S

Feghali, C.A., K.L. Bost, D.W. Boulware and L.S. Levy, 1994. Control of IL-6 expression and response in fibroblasts from patients with systemic sclerosis. Autoimmunity, 17: 309-318. DOI: $10.3109 / 08916939409010671$

Fineschi, S., L. Goffin, R. Rezzonico, F. Cozzi and J.M. Dayer et al., 2008. Antifibroblast antibodies in systemic sclerosis induce fibroblasts to produce profibrotic chemokines, with partial exploitation of toll-like receptor 4. Arthritis Rheumatism, 58: 3913-3923. DOI: 10.1002/art.24049

Fleischmajer, R., J. Perlish and J. Reeves, 1977. Cellular infiltrates in scleroderma skin. Arthritis Rheumatism, 20: 975-984. DOI: $10.1002 /$ art. 1780200410

Hua, J., K. Kirou, C. Lee and M.K. Crow, 2006. Functional assay of type I interferon in systemic lupus erythematosus plasma and association with anti-RNA binding protein autoantibodies. Arthritis Rheumatism, 54: 1906-1916.

DOI: $10.1002 /$ art.21890

Janeway Jr, C.A., 1992. The immune system evolved to discriminate infectious nonself from noninfectious self. Immunol. Today, 13: 11-16.

DOI: 10.1016/0167-5699(92)90198-G

Jimenez, S.A. and C.T. Derk, 2004. Following the molecular pathways toward an understanding of the pathogenesis of systemic sclerosis. Ann. Internal Med., 140: 37-50.

DOI: 10.7326/0003-4819-140-2-200401200-00013

Kissin, E.Y., P.A. Merkel and R. Lafyatis, 2006. Myofibroblasts and hyalinized collagen as markers of skin disease in systemic sclerosis. Arthritis Rheumatism, 54: 3655-3660.

DOI: $10.1002 / \operatorname{art} .22186$

LeRoy, E.C., C. Black, R. Fleischmajer, S. Jablonska and T. Krieg et al., 1988. Scleroderma (systemic sclerosis): Classification, subsets and pathogenesis. J. Rheumatol., 15: 202-205. PMID: 3361530

Mathai, S.K., M. Gulati, X. Peng, T.R. Russell and A.C. Shaw et al., 2010. Circulating monocytes from systemic sclerosis patients with interstitial lung disease show an enhanced profibrotic phenotype. Laboratory Investigat., 90: 812-823.

DOI: $10.1038 /$ labinvest.2010.73
Pattanaik, D., M. Brown, B.C. Postlethwaite and A.E. Postlethwaite, 2015. Pathogenesis of systemic sclerosis. Frontiers Immunol., 6: 272-272.

DOI: $10.3389 /$ fimmu.2015.00272

Roelofs, M., L. Joosten, S. Abdollahi-Roodsaz, A. Van Lieshout and T. Sprong et al., 2005. The expression of toll-like receptors 3 and 7 in rheumatoid arthritis synovium is increased and costimulation of toll-like receptors 3,4 and $7 / 8$ results in synergistic cytokine production by dendritic cells. Arthritis Rheumatism, 52: 2313-2322.

DOI: $10.1002 /$ art.21278

Sakoguchi, A., W. Nakayama, M. Jinnin, Z. Wang and K. Yamane et al., 2013. The expression profile of the toll-like receptor family in scleroderma dermal fibroblasts. Clin. Exp. Rheumatol., 32: 4-9. PMID: 24959869

Schmittgen, T.D. and K.J. Livak, 2008. Analyzing realtime PCR data by the comparative CT method. Nature Protocols, 3: 1101-1108. DOI: $10.1038 /$ nprot.2008.73

Takeda, K. and S. Akira, 2004. TLR signaling pathways. Seminars Immunol., 16: 3-9. DOI: $10.1016 /$ j.smim.2003.10.003

Tan, F., X. Zhou, M. Mayes, P. Gourh and X. Guo et al., 2006. Signatures of differentially regulated interferon gene expression and vasculotrophism in the peripheral blood cells of systemic sclerosis patients. Rheumatology, 45: 694-702. DOI: $10.1093 /$ rheumatology/kei244

Tan, F.K. and F.C. Arnett, 2000. Genetic factors in the etiology of systemic sclerosis and Raynaud phenomenon. Current Opin. Rheumatol., 12: 511-519. DOI: 10.1097/00002281-200011000-00007

Tomasek, J.J., G. Gabbiani, B. Hinz, C. Chaponnier and R.A. Brown, 2002. Myofibroblasts and mechanoregulation of connective tissue remodelling. Nature Rev. Molecular Cell Biol., 3: 349-363. DOI: $10.1038 / \mathrm{nrm} 809$

van Lieshout, A., M. Vonk, S. Bredie, L. Joosten and M. Netea et al., 2009. Enhanced interleukin-10 production by dendritic cells upon stimulation with Toll-like receptor 4 agonists in systemic sclerosis that is possibly implicated in CCL18 secretion. Scandinavian J. Rheumatol., 38: 282-290. DOI: $10.1080 / 03009740802572467$

Varga, J. and D. Abraham, 2007. Systemic sclerosis: A prototypic multisystem fibrotic disorder. J. Clin. Investigat., 117: 557-567. DOI: 10.1172/JCI31139

Varga, J., 2007. Systemic sclerosis: An update. Bull. NYU Hospital Joint Dis., 66: 198-202. PMID: 18937632 